\title{
Proposta do uso de pool de sangue total como controle interno de qualidade em hematologia
}

\author{
Proposal for the use of a pool of whole blood as internal quality control in hematology
}

Carina Daniele Schons'; Rejane Giacomelli Tavares²

\begin{tabular}{|c|c|}
\hline unitermos & resumo \\
\hline $\begin{array}{l}\text { Controle de qualidade } \\
\text { Controle interno } \\
\text { Pool } \\
\text { Hematologia }\end{array}$ & $\begin{array}{l}\text { Introdução: A confiabilidade dos resultados do laboratório é garantida pela realização do controle } \\
\text { de qualidade, que tem como funções básicas análise, pesquisa e prevenção da ocorrência de erros } \\
\text { laboratoriais por meio de programas que abrangem tanto o controle interno quanto o externo. Objetivo: } \\
\text { Propor a padronização de utilização de pool de sangue total como controle interno de qualidade no } \\
\text { setor de hematologia. Método: Foram selecionadas amostras de sangue total, colhidas com ácido } \\
\text { etilenodiaminotetracético (EDTA), de mesmos grupo sanguíneo e fator Rh, livres de interferentes, } \\
\text { como hemólise, lipemia e icterícia. De um total de } 30 \mathrm{ml} \text { de sangue total, obtiveram-se três alíquotas } \\
\text { de } 10 \mathrm{ml} \text { cada, às quais foram adicionados, respectivamente, } 0 \mathrm{ml} \text { (sem adição), } 1 \mathrm{ml} \text { e } 5 \mathrm{ml} \text { de glicerol } \\
\text { (conservante). As amostras foram avaliadas em contador automático ADVIA }{ }^{\circledR} 60 \text {. Após determinação dos } \\
\text { valores de média e DP, todas as amostras foram avaliadas por um período de } 45 \text { dias úteis para confecção } \\
\text { do gráfico de Levey-Jennings e verificação da estabilidade da amostra. Resultado e conclusão: Podemos } \\
\text { verificar que o pool de sangue total, preparado de acordo com a metodologia proposta, não apresenta } \\
\text { estabilidade necessária para sua utilização, como controle interno alternativo no setor de hematologia. }\end{array}$ \\
\hline
\end{tabular}

abstract

Introduction: The reliability of laboratory results is ensured by the implementation of quality control, which has basic functions, such as analysis, research and prevention of laboratory errors through programs that encompass both internal and external control. Objective: To propose a standard method to use pooled whole blood as internal quality control in the Hematology division. Method: The selected whole blood samples were collected with EDTA, belonged to the same blood group and Rh factor and did not present interfering factors, such as hemolysis, lipemia and icterus. From a total of $30 \mathrm{ml}$ of whole blood it was obtained 3 aliquots of $10 \mathrm{ml}$ each, to which $0 \mathrm{ml}$ (no addition), $1 \mathrm{ml}$ and $5 \mathrm{ml}$ of glycerol (preservative) were added, respectively. The samples were analyzed in the automatic counter $A D V I A^{\circledR} 60$. After the determination of mean and standard deviation values of all samples, they were evaluated for a period of 45 working days in order to produce a Levey-Jennings graph and to check their stability. Result and conclusion: We could verify that the pool of whole blood prepared in accordance with the methodology proposed does not present the required stability to be used as an alternative internal control in the Hematology division. key words

Quality control

Internal control

Pool

Hematology 


\section{Introdução}

A qualidade é definida como a conformidade às necessidades daqueles que fazem uso do produto ou serviço. Mais diretamente, a qualidade deve ser referida à satisfação das necessidades e das expectativas dos clientes, usuários e empresas de prestação de serviços $(5,15,9)$

Como importante benefício da melhoria da qualidade podem-se citar a redução do desperdício e a elevação da produtividade, a qual, por sua vez, reduz custos e fornece uma vantagem competitiva ${ }^{(5)}$. Nos laboratórios clínicos, melhorias na qualidade podem ser comprovadas pela diminuição de repetições de exames, implicando, ainda, maior agilidade no tempo útil para entrega dos resultados e maior confiabilidade do corpo clínico e dos clientes diretos ${ }^{(1)}$.

Este padrão de qualidade pode ser obtido pela utilização dos princípios de controle de qualidade, com utilização de controles de qualidade comerciais e internos alternativos, bem como a participação em programas de controle de qualidade externos. Além disso, muitas decisões tomadas com base nos resultados de controle de qualidade podem implicar maior credibilidade do laboratório(10).

$\mathrm{O}$ controle interno de qualidade (CIQ) tem a finalidade de verificar a calibração dos sistemas analíticos, garantir a reprodutibilidade (precisão) dos resultados, além de indicar a necessidade de ações corretivas. Todo laboratório precisa estabelecer e sustentar um sistema próprio de melhoria na qualidade, considerando tipo, complexidade dos exames que realiza e volume. Em hematologia, a utilização de CQI se torna mais crítica pela rápida perda da estabilidade dos materiais de controle utilizados, o que torna o ciclo de acompanhamento de um lote mais curto $^{(9)}$.

Para implementar um controle interno eficiente, o primeiro passo é a seleção do controle a ser utilizado. Atualmente, a melhor opção é a utilização de soluções comerciais, como determina a Agência Nacional de Vigilância Sanitária (ANVISA) na Resolução RDC 302/2005 para funcionamento de laboratórios clínicos. O regulamento deixa claro que alguma forma de controle deve existir e que o laboratório deve buscar controles comerciais padronizados. Esta mesma resolução permite que, na indisponibilidade de controles comerciais adequados, podem-se adotar formas alternativas de controle que permitam avaliar a precisão da análise. Pode-se citar como exemplo a utilização de pool de soros ${ }^{(3)}$. Esse procedimento alternativo já é padronizado e bastante utilizado em setores como bioquímica e imunologia ${ }^{(5)}$.
$\mathrm{Na}$ hematologia destacam-se unicamente os controles comerciais, o que dificulta muitas vezes a diminuição dos custos. Algumas alternativas constituem-se em utilização do algoritmo de Bull, repetição de amostras de paciente - para os parâmetros globais - e comparação de leitura microscópica para o diferencial de células.

O algoritmo de Bull monitora, ao longo do dia, as médias de resultados de pacientes. Este é um método antigo que consiste em comparar as médias obtidas a cada 20 valores consecutivos processados, com o valor médio acumulado. Muitos equipamentos já realizam este cálculo automaticamente. Quanto maior o número de pacientes/dia, mais consistentes são os resultados do método. As principais limitações deste método são verificadas em laboratórios com volume reduzido de análise e de atendimento hospitalar, que podem apresentar grande oscilação dos resultados devido à população atendida(7, 12).

Uma segunda opção é a repetição de amostras de pacientes. Neste caso, monitora-se a diferença entre resultados de uma mesma amostra quando dosada em diferentes momentos, como descrito na norma Calibration and Quality Control of Automated Hematology Analizers H38P (CLSI), que recomenda que esta repetição seja feita dentro de uma mesma corrida. Como a estabilidade esperada para amostras de paciente é de 24 horas, o laboratório deve selecionar minimamente cinco pacientes durante um dia e testar novamente na manhã seguinte. Após obtenção dos resultados, para cada amostra deve-se calcular a diferença entre os resultados inicial e final para, então, obterem-se os valores de média, desvio padrão (DP) e coeficiente de variação das diferenças acumuladas para cada grupo de pacientes testado. Isso permitirá que o laboratório conheça a variação média do seu processo ao longo do tempo ${ }^{(12)}$.

É interessante observar que o CLSI na norma Assessment of Laboratory Tests when Proficiency Testing is not Avaiable GP29 menciona que este método - denominado "arraste de amostras" - era muito utilizado até meados de 1970, quando se passou a adotar o método de Bull. Esta opção ganha importância especialmente no Brasil, onde muitos laboratórios ainda possuem equipamentos que não fornecem o algoritmo de Bull(12).

Para a contagem diferencial, a norma CLSI H2O Reference Leukocyte Differential Count Proportional and Evaluation of Instrumental Methods menciona como formas de controle as comparações entre observadores e entre observadores e analisador. Para as duas comparações podem-se selecionar amostras de pacientes com diferentes perfis de resultados, sendo que o laboratório deve definir 
a variação aceitável entre os mesmos. Para a comparação interobservadores podem-se ainda utilizar materiais de ensaio de proficiência, para os quais a faixa de leitura aceitável já foi definida. Quanto maiores a frequência e o volume de materiais analisados, mais eficiente será o controle ${ }^{(12,14)}$.

Embora órgãos nacionais e internacionais permitam a utilização dessas formas alternativas de controle de qualidade, é importante ressaltar que sua padronização deve iniciar-se ainda quando o laboratório dispõe de controle comercial, garantindo, assim, que a variação do processo encontrada represente a realidade da análise sob controle, ou seja, a partir desses dados poder-se-á definir a variação aceitável e identificar quando a mesma estiver fora de controle ${ }^{(7,12)}$. Diante da escassez de métodos de controle de qualidade alternativos padronizados e aceitos, este trabalho buscou avaliar a estabilidade de um pool de sangue total, cuja utilização contribuiria para a monitoração da precisão dos equipamentos, com possível redução dos custos vigentes no laboratório.

\section{Objetivo}

Verificar a possibilidade de utilização de pool de sangue total como controle interno alternativo no setor de hematologia por meio da avaliação diária do mesmo para acompanhamento da estabilidade dos parâmetros hematócrito, hemoglobina, contagem total de hemácias, contagem total de leucócitos e contagem total de plaquetas.

\section{Material e método}

Para a confecção do pool de sangue total foram selecionadas 10 amostras colhidas com ácido etilenodiaminotetracético (EDTA), de mesmos grupo sanguíneo e fator Rh, livres de interferentes como hemólise, lipemia e icterícia. A partir do volume total obtido da mistura das amostras, obtiveram-se três alíquotas de $10 \mathrm{ml}$ cada. A alíquota 1 foi constituída de sangue total homogeneizado, sem adição de conservantes. À alíquota 2 foi adicionado $1 \mathrm{ml}$ de glicerol (conservante) e à alíquota $3,5 \mathrm{ml}$ de glicerol. Embora se tenha obtido uma diluição importante no pool número 3 , a mesma não interferiu na avaliação da estabilidade dos componentes.

As amostras foram avaliadas no Setor de Hematologia do Laboratório de Biomedicina da Federação de Estabelecimento de Ensino Superior em Novo Hamburgo (FEEVALE), em contador automático $A D V I A^{\circledR} 60$. Após determinação dos valores de média e DP, foi realizada a avaliação de cada uma das amostras (conservante $0 \mathrm{ml}, 1 \mathrm{ml} \mathrm{e} 5 \mathrm{ml}$ ), em um período de 45 dias úteis, para confecção do gráfico de Levey-Jennings e verificação da estabilidade de alguns componentes da amostra.

\section{Resultados}

Inicialmente foram determinados os valores de média e DP dos pools 1, 2 e 3, contendo, respectivamente, $0,1 \mathrm{ml}$ ou $5 \mathrm{ml}$ de glicerol. A partir desses dados foi possível elaborar gráficos de Levey-Jennings. Nas Figuras 1A, 1B e 1C estão plotados os dados diários representativos para células vermelhas, utilizando-se média e DP calculados com dados de 20 valores obtidos em dias diferentes e consecutivos. A diminuição observada nos valores de média é decorrente da diluição da amostra, em função da adição do conservante glicerol, o que, entretanto, não impede a avaliação

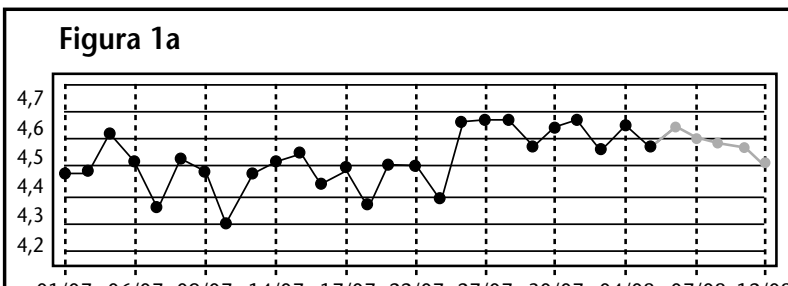

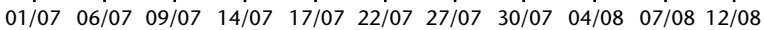

Figura $1 \mathrm{~b}$

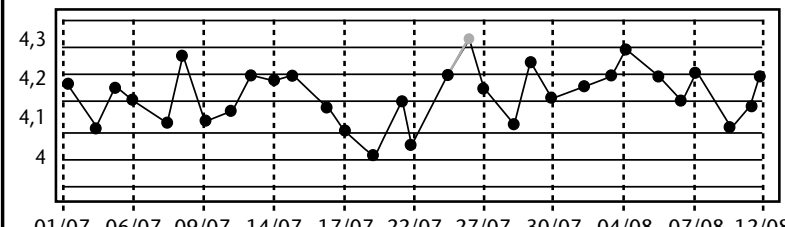

$\begin{array}{lllllllllllllllllll}01 / 07 & 06 / 07 & 09 / 07 & 14 / 07 & 17 / 07 & 22 / 07 & 27 / 07 & 30 / 07 & 04 / 08 & 07 / 08 & 12 / 08\end{array}$

Figura 1c

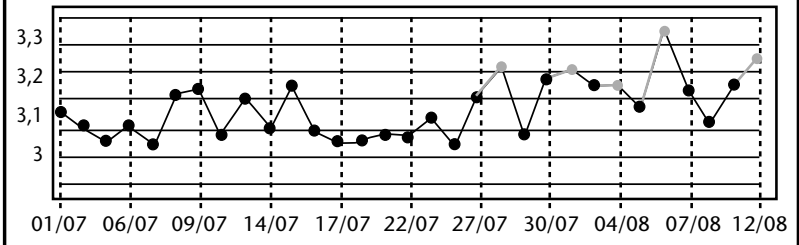

Figura 1 - Gráfico de Levey-Jennings representativo para a contagem de células vermelhas. Os dados plotados em preto encontram-se nos valores limite aceitáveis. Os dados plotados em cinza representam erros segundo as regras múltiplas de Westgard $(\mathrm{n}=42)$. Dados são expressos em média \pm desvio padrão. (A) Sem adição de glicerol $(4,47 \pm 0,09)$; (B) adição de 1 $\mathrm{ml}$ de glicerol (10\%) (4,14 \pm 0,07); (C) adição de $5 \mathrm{ml}$ de glicerol (50\%) $(3,08 \pm 0,05)$ 
da estabilidade do analito. Quando avaliada a estabilidade das células brancas, independentemente do uso ou não de conservante, observou-se que a mesma é bastante baixa, em torno de sete a 10 dias (Figuras $\mathbf{2 A}, \mathbf{2 B}$ e $\mathbf{2 C}$ ).

Em relação ao uso do conservante glicerol, nossos dados demonstraram que tanto a utilização de $1 \mathrm{ml}$ (10\%) quanto a de $5 \mathrm{ml}(50 \%)$ permitiram maior tempo de conservação dos índices hematimétricos (hematócrito, hemoglobina, células vermelhas e plaquetas) quando se utilizam para cálculo de valor médio os dados obtidos em 20 dias consecutivos (dados não mostrados).

\section{Discussão}

A necessidade de controle de qualidade laboratorial é uma exigência cada vez maior em todos os serviços prestados, incluindo os laboratórios clínicos. Nestes, a primeira iniciativa interlaboratorial de controle da qualidade foi realizada nos Estados Unidos, em 1947, por Belk e Sunderman. Eles empregaram um pool de soro humano para comparar as análises de um grupo de laboratórios. Em 1950, Levey e Jennings aprimoraram o controle interno, já praticado na

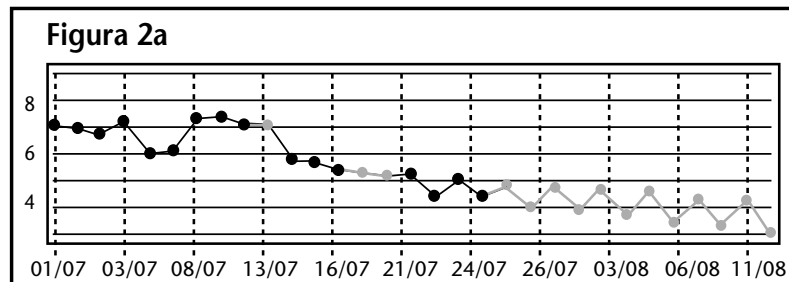

Figura $2 b$

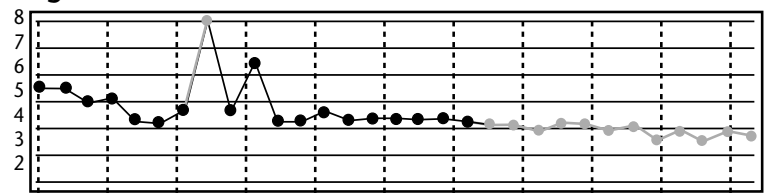

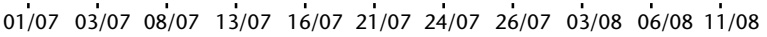

Figura 2c

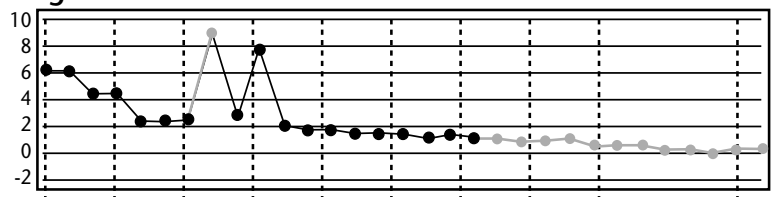

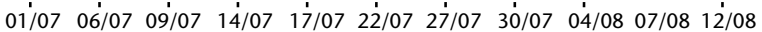

Figura 2 - Gráfico de Levey-Jennings representativo para a contagem de células brancas. Os dados plotados em preto encontram-se nos valores limite aceitáveis. Os dados plotados em cinza representam erros segundo as regras múltiplas de Westgard $(\mathrm{n}=42)$. Dados são expressos em média \pm desvio padrão. (A) Sem adição de glicerol $(5,88 \pm 1,14)$; (B) adição de 1 $\mathrm{ml}$ de glicerol (10\%) (4,42 \pm 0,99); (C) adição de $5 \mathrm{ml}$ de glicerol (50\%) $(4,21 \pm 2,02)$ época, por meio da representação gráfica dos valores de cada dia. Essas atividades foram denominadas de Programas de Controle de Qualidade, que atualmente incluem as atividades de controles externo e interno de qualidade ${ }^{(9)}$.

Segundo a RDC 302, o laboratório clínico deve realizar o controle interno da qualidade contemplando o monitoramento do processo analítico das amostras controle comerciais, regularizadas junto à ANVISA/MS, com registro dos resultados obtidos e análise dos dados, além da utilização de formas alternativas de controle interno que permitam a avaliação da precisão do sistema analítico ${ }^{(3)}$.

Com base nesta resolução, cada vez mais se têm buscado formas que sejam econômicas e confiáveis para o acompanhamento interno da qualidade. Como uma possibilidade surge a utilização de misturas de amostras obtidas no próprio laboratório, conhecidas como pool. Essa alternativa já é padronizada e bastante utilizada quando nos referimos ao uso de misturas de soros, utilizadas nos setores de bioquímica e imunologia. Para os demais setores, especialmente a hematologia, tal procedimento ainda carece de padronização e estudo de métodos adequados de conservação dos constituintes sanguíneos, fato refletido pela escassez de artigos científicos relacionados com o assunto.

Embora este método seja indicado somente para a aferição da precisão da metodologia utilizada, e não da exatidão, é necessário que os parâmetros estatísticos de média e DP sejam determinados para posterior construção dos gráficos de Levey-Jennings.

No momento da padronização dos valores de média e DP é importante tomar cuidado para evitar a interferência de variáveis pré-analíticas (coleta das amostras), analíticas (variações do equipamento utilizado ou troca de reagentes) ou, ainda, operacionais (homogeneização das amostras, temperatura da amostra, tempo decorrido entre a coleta e a análise ou tempo de conservação em geladeira). Para isso o procedimento ideal de determinação desses valores é a utilização de 20 valores, obtidos em dias diferentes(3). Esse procedimento é bastante facilitado quando se trabalha com amostras mais estáveis, como o soro, por exemplo.

Para as amostras utilizadas na hematologia (sangue total anticoagulado com EDTA), esse procedimento apresenta como principal limitante o tempo de estabilidade da amostra. Segundo Simon et al. ${ }^{(16)}$, as hemácias apresentam aumento da suscetibilidade à lise após 24 horas em contato com EDTA. Assim, cuidado adicional também deve ser tomado para que os possíveis conservantes utilizados sejam adicionados no menor tempo possível após a obtenção das amostras. 
Em nosso estudo, os valores de média e DP foram determinados de duas formas diferentes, para efeitos de comparação: em um primeiro momento, os 20 valores foram determinados em quatro dias consecutivos (cinco dosagens a cada dia) e, em um segundo momento utilizaram-se os 20 primeiros valores obtidos (efetivamente em dias diferentes). Nossos dados demonstraram que a utilização dos valores obtidos em 20 dias diferentes permite uma análise mais adequada dos dados, visto que os valores posteriormente obtidos apresentam-se dentro dos limites permitidos de variação por um período aproximado de 15 dias, exceto para a contagem global de leucócitos (Figuras 1A, B, C e 2 A, B, C). Esta última possui estabilidade aproximada de sete dias apenas. Este achado é compatível com outros estudos que demonstram que o sangue total armazenado por 24 horas mantém pequena quantidade de leucócitos viáveis(13).

Ainda em relação aos leucócitos, observamos que, além da grande diminuição na contagem total, ocorreram também variações importantes na contagem diferencial, sendo que a contagem de linfócitos era exacerbada com o decorrer do tempo. Buscando entender esse fato, foram avaliados esfregaços corados das amostras, nos quais se pode comprovar que grande parte das células brancas estava lisada e o núcleo resultante apresentava-se bastante deformado e involuído, assumindo o aspecto morfológico do leucócito mononuclear linfócito.

A definição dos valores de média e DP utilizando-se os valores obtidos com menor tempo (quatro dias, cinco dosagens diárias) se mostrou bastante inadequada, pois observamos importante queda dos valores posteriores, com extrapolação dos valores máximos permitidos (média \pm 3 DP), independentemente da utilização de conservante (dados não mostrados). Esse fato nos permite sugerir que o glicerol não possua atividade adequada de conservação das células nos primeiros dias de armazenamento das amostras, parecendo exercer papel mais efetivo em estocagem mais prolongada, ou seja, decorridos sete dias.

O glicerol (1, 2, 3 propanotriol ou glicerina) foi escolhido como conservante a ser testado neste estudo por não apresentar toxicidade, cor ou odor, além de ser um agente crioprotetor e osmorregulador, diminuindo a permeabilidade da membrana e contribuindo para a manutenção da atividade celular ${ }^{(4)}$. Também é um importante intermediário do processo de gliconeogênese, em que funciona como fonte de carbono ${ }^{(8)}$, sendo utilizado em bancos de sangue como agente conservante de hemácias ${ }^{(2,13)}$.

Em relação à quantificação de plaquetas, observamos grande variação entre as dosagens, refletida por um valor bastante alto de desvio padrão. De acordo com Hauser et $a{ }^{(7)}$, tal achado é explicado pelo maior número de variáveis interferentes na precisão da contagem de plaquetas, como, por exemplo, a variação no tamanho das plaquetas, o satelitismo plaquetário ou a presença de agregados plaquetários, de fragmentos de leucócitos ou eritrócitos ou mesmo de partículas estranhas. Somam-se a isso as variações relativas ao equipamento analítico utilizado, relacionadas com instabilidades das medidas decorrente de ruídos na corrente elétrica ou manutenção inadequada do mesmo.

\section{Conclusão}

A partir dos resultados obtidos neste trabalho pode-se afirmar que o pool de sangue total utilizando-se adição de $1 \mathrm{ml}$ ou $5 \mathrm{ml}$ de glicerol como conservante apresenta reduzido tempo de estabilidade, sendo de aproximadamente 15 dias para série vermelha e plaquetas e sete dias para série branca. Assim, o mesmo não é recomendado para utilização diária como controle interno alternativo no setor de hematologia, ressaltando-se que cada laboratório deve realizar a avaliação custo-benefício do processo para que este procedimento seja incorporado à rotina analítica. Adicionalmente, estudos com outros conservantes já utilizados na hemoterapia, como citrato-fosfato-dextrose (CPD) e citrato-fosfato-dextrose-adenina (CPDA-1), devem ser realizados para possível padronização de um pool de sangue total.

Independentemente da utilização de outras formas de controle interno alternativo, é fundamental que a primeira escolha do laboratório seja a utilização de controles comerciais, que possuem maior estabilidade e permitem a análise do material por um período maior.

\section{Referências}

1. ABREU, R. C. L. CCQ - círculos de controle da qualidade: a integração trabalho homem-qualidade total. 2. ed. Rio de Janeiro: Qualitymark, 1993.

2. ANVISA. ResoluçãoRDCn゚ 153, de14dejunhode2004. Disponível em: <http://www.bancodesangue.com.br/website/content/ bancosangue/geral/index. php?idPagina=HOSPITAL LEGISLACAO\&idMenu=MENU_EUSOU_HOSPITAL $>$.

3. ANVISA. Resolução RE no 302, de 13 de outubro de 2005. Disponível em: <http://e-legis.anvisa.gov.br/leisr. php?id=19176> 
4. ARRUDA, P. V.; RODRIGUES, R. C. L. B.; FELIPE, M. G. A. Glicerol: um subproduto com grande capacidade industrial e metabólica. Rev Analytica, n. 26, p. 56-62, 2007.

5. BURTIZ, C. A.; ASHWOOD, E. R. (orgs). TIETZ: fundamentos de química clínica. 4. ed. Rio de Janeiro: Guanabara Koogan, 1998.

6. FERREIRA, M. F. R.; VIEIRA, L. M. F.; BASTOS, M. Garantia da qualidade do hemograma automatizado. Rev Bras Anal Clin, v. 34, n. 3. p. 121-9, 2002.

7. HAUSER, A. B. et al. Programa de controle da qualidade externo em hematologia: variações interlaboratoriais para eritrograma e plaquetas em Curitiba e Região Metropolitana, PR. Rev Bras Anal Clin, v. 36, n. 3, p. 155-8, 2004.

8. LEHNINGER, A. L. Bioquímica. 1. ed. São Paulo: Edgard Blücher, 2000.

9. LOPES, H. J. J. Garantia e controle da qualidade no laboratório clínico. Analisa, 2003. Disponível em: <hppt://www.goldanalisa.com.br/publicacoes/ Garantia_e_Controle_da_Qualidade_no_Laboratorio_ Clinico.pdf>.
10. MANUAL DO PNCQ. Disponível em: <hppt://www.sbpc. org.br/files/pdf/manualdolaboratorio>.

11. MORRISON, L. R. Glycerol. Encyclopedia of Chemical Technology. New York: Wiley, 1994. p. 921-32.

12. OLIVEIRA, D. Controle interno alternativo para hematologia. Boletim Qualifique, v. 22, p. 1-2, 2008.

13. RAZOUK, F. H.; REICHE, E. M. V. Caracterização, produção e indicação clínica dos principais hemocomponentes. Rev Bras Hematol Hemoter, v. 26, n. 2, p. 126-34, 2004.

14. SAXENA, R. et al. Impact of external haematology proficiency testing on quality of laboratories. Indian J Med Res, v. 126, p. 428-32, 2007.

15. SILOAHO, M.; PUHAKAINEN, E. Implementation of a quality system in a clinical laboratory: evaluation of quality indicators. Accred Qual Assur, v. 5, n. 5, p. 182-90, 2000.

16. SIMON, C. F. et al. Patologia clínica: colheita, conservação e remessa de amostras. Veterinária em Foco, v. 4, n. 2, p. 131-41, 2007. 